\title{
Approximate closed-form solution for solar sail spiral trajectories with sail degradation
}

\author{
Colin R Mclnnes ${ }^{1}$ \\ University of Strathclyde, Glasgow G1 1XJ, UK
}

\section{Introduction}

Solar sails have long been considered as a means of enabling new high-energy missions, such as solar polar orbiter, planetary sample return and heliopause probes, along with families of highly nonKeplerian orbits for space weather and Earth observation missions, as reviewed in Refs 1 and 2. While most prior analysis of solar sail orbital dynamics assumes that the optical properties of the sail are time-invariant, it is expected that the sail membrane will slowly degrade due to cumulative, longterm exposure to solar radiation. The effect of such degradation was investigated in some detail by Dachwald and co-workers through a combination of modelling and numerical optimization. ${ }^{3,4}$ As expected, it was found that degradation has an impact on the trip time of solar sails to target orbits and the ability of solar sails to realize non-Keplerian orbits.

In this Note, an approximate closed-form solution is presented for solar sail spiral trajectories with sail degradation. Since exposure to the space environment is cumulative, the impact of degradation on the sail thrust magnitude forms an integral function over the spiral duration. The time evolution of the solar sail spiral trajectory is therefore described by an integro-differential equation, which for this problem does not apparently possess an explicit closed-form solution. However, it is possible to obtain an implicit solution to the problem which provides an approximate representation of the evolution of the solar sail spiral trajectory. This allows an initial estimation of the impact of sail degradation on mission performance to be made.

\footnotetext{
${ }^{1}$ Director, Advanced Space Concepts Laboratory, Department of Mechanical and Aerospace Engineering, colin.mcinnes@strath.ac.uk
} 
Limits are also found which bound the motion of the solar sail to an annulus, whose inner and outer radii are defined by the sail degradation rate. These limits correspond to the asymptotic behaviour of the solar sail where the sail becomes completely absorbing, the transverse component of sail thrust vanishes and the spiral terminates. In addition, it is shown that the optimal fixed sail pitch angle required to maximize the inner and outer radius of the annulus differs from the usual sail pitch angle which maximizes the transverse component of sail thrust. For a solar sail subject to degradation, the optimum fixed sail pitch angle must trade-off maximising the transverse component of sail thrust while minimising the projected sail area exposed to solar radiation.

Finally, while these asymptotic limits of motion represent an operational constraint, they could in principle be used as a means of enabling entirely passive orbit transfer. For example, a small, low cost solar sail with a passively fixed pitch angle could deliver a payload onto a quasi-circular spiral trajectory. If the sail film is engineered to degrade at a certain rate, the asymptotic orbit the sail winds onto can then be chosen a priori. The solar sail will then perform a near circle-to-circle transfer, in principle satisfying a simple two-point boundary value problem, but using only a passively fixed sail pitch angle. Such a mode of transfer could be used to deliver payloads to given heliocentric orbits, for example for space physics applications.

\section{Sail degradation model}

A simple optical model of a solar sail will now be defined with time-varying, non-perfect reflectivity $\eta(t)$. Following Dachwald et. al., ${ }^{3,4}$ the solar radiation pressure induced acceleration $\overrightarrow{\boldsymbol{a}}$ acting on the sail will be written in terms of radial $\overrightarrow{\boldsymbol{r}}$ and transverse components $\overrightarrow{\boldsymbol{t}}$ as

$$
\overrightarrow{\boldsymbol{a}}=\frac{P A}{m} \eta(t) \cos (\alpha)(1+\eta(t) \cos (2 \alpha)) \overrightarrow{\boldsymbol{r}}+\frac{P A}{m} \eta(t) \cos (\alpha) \sin (2 \alpha) \overrightarrow{\boldsymbol{t}}
$$

where $P$ is the solar radiation pressure, $A$ is the sail area, $m$ is the sail mass and $\alpha$ is the sail pitch angle relative to the Sun-line, as shown in Fig. 1 . It can be seen that as the sail film degrades and 
$\eta(t) \rightarrow 0$ the transverse component of sail acceleration vanishes, leaving only a radial acceleration due to the now fully absorbing sail surface. In this limit the sail spiral motion will terminate and the sail will asymptotically approach a Keplerian orbit, modified by the remaining inverse square radial acceleration due to solar radiation pressure.

Again following Dachwald et. al., ${ }^{3,4}$ it will be assumed that the degradation of the sail reflectivity scales with the absorbed flux of solar radiation. Therefore, the time evolution of the solar sail reflectivity will be described as

$$
\frac{d \eta(t)}{d t}=-\varepsilon\left(\frac{R}{r(t)}\right)^{2} \cos (\alpha) \eta(t)
$$

where $r(t)$ is the solar sail orbit radius, $R$ is a reference distance (taken as 1 astronomical unit (AU)) and $\varepsilon$ is a sail degradation parameter which defines the rate of degradation of the sail reflectivity due to exposure to solar radiation. Integrating Eq. (2) the sail reflectivity therefore evolves as

$$
\eta(t)=\eta_{O} \exp \left(-\varepsilon \int_{0}^{t}\left(\frac{R}{r\left(t^{\prime}\right)}\right)^{2} \cos (\alpha) d t^{\prime}\right)
$$

for some initial reflectivity $\eta_{O}$, later assumed to be unity. In order to provide a meaningful way of representing the sail degradation parameter $\varepsilon$, it is assumed that the solar sail is fixed at $1 \mathrm{AU}$ $(r(t)=R)$, facing the Sun $(\alpha=0)$ so that Eq. (3) integrates directly to

$$
\eta(t)=\eta_{O} \exp (-\varepsilon t)
$$

Again following Dachwald et. al., ${ }^{3,4}$ the sail degradation half-life $T$ can be defined as the time required for the sail reflectivity to fall to half its initial value, such that $\eta(\tau)=\eta_{O} / 2$. Assuming that the sail initially has ideal reflectivity such that $\eta_{O}=1$, the sail degradation parameter $\varepsilon$ can be written simply as

$$
\varepsilon=\frac{\log (2)}{\mathrm{T}}
$$


Therefore, using the sail degradation half-life $T$ as a performance metric, the sail degradation model is now fully defined. Using this simple model for the sail degradation, the approximate evolution of the solar sail spiral trajectory can be investigated.

\section{Approximate closed-form solution}

Time-invariant optical properties

Now that the sail degradation model has been defined, the influence of degradation on the orbit evolution of a solar sail will be considered. In order to provide a simple, closed-form solution it will firstly be assumed that the solar sail orbit is a quasi-circular spiral with a fixed sail pitch angle. Then, the evolution of the sail orbit radius can be determined from the rate of change of orbit energy $E$ as

$$
\frac{d E}{d t}=\overrightarrow{\boldsymbol{a}} \cdot \overrightarrow{\boldsymbol{v}}
$$

where the two-body orbit energy is defined by $E=-\mu / 2 r(t)$ for orbit radius $r(t)$ and gravitational parameter $\mu$, and where the sail acceleration $\overrightarrow{\boldsymbol{a}}$ is defined by Eq. (1). For a quasi-circular orbit, the solar sail velocity vector is defined by $\overrightarrow{\mathbf{v}}=\sqrt{\mu / r(t)} \overrightarrow{\mathbf{t}}$ so that

$$
\frac{d E}{d t}=\frac{2 P A}{m} \eta(t) \cos ^{2}(\alpha) \sin (\alpha) \sqrt{\frac{\mu}{r(t)}}
$$

where $2 P A / m$ is the solar radiation pressure induced acceleration experienced by the solar sail. The solar radiation pressure scales as the inverse square of the solar sail orbit radius such that $P=\tilde{P}(R / r(t))^{2}$, where $\tilde{P}$ is the solar radiation pressure at distance $R$.

The sail lightness number is now defined as the ratio of the solar radiation pressure induced acceleration to the solar gravitational acceleration experienced by the solar sail. ${ }^{5}$ Since both solar gravity and solar radiation pressure scale as the inverse square of the sail orbit radius $r(t)$, the lightness number can be defined as $\beta=2 \widetilde{P} A R^{2} / m \mu$. 
In order to proceed, the problem can now be scaled through the definition of a new dimensionless dependant variable $\xi(t)=r(t) / R$, and a new independent variable $\tau=\omega t$, where $\omega=\sqrt{\mu / R^{3}}$. Then, substituting for the two-body orbit energy $E$ in Eq. (7) it can be shown that

$$
\frac{\mu}{2 \xi(\tau)^{2}} \frac{d \xi(\tau)}{d \tau}=\beta \frac{\mu}{\xi(\tau)^{2}} \eta(\tau) \cos ^{2}(\alpha) \sin (\alpha) \xi(\tau)^{-1 / 2}
$$

so that the sail orbit radius evolves as

$$
\frac{d \xi(\tau)}{d \tau}=2 \beta \eta(\tau) \cos ^{2}(\alpha) \sin (\alpha) \xi(\tau)^{-1 / 2}
$$

For a sail with time-invariant optical properties such that $\eta(t)=1$, and assuming that the initial orbit of the solar sail is at $1 \mathrm{AU}$, such that $\xi(0)=1$, Eq. (9) can be integrated directly to obtain the sail orbit radius as

$$
\xi(\tau)=\left(1+3 \beta \cos ^{2}(\alpha) \sin (\alpha) \tau\right)^{2 / 3}
$$

so that the orbit radius scales as $\xi(\tau) \sim \tau^{2 / 3}$. The sail polar angle $\theta(\tau)$ can then be obtained from

$$
\frac{d \theta(\tau)}{d \tau}=\xi(t)^{-3 / 2}
$$

which integrates directly to yield

$$
\theta(\tau)=\frac{1}{3 \beta \cos ^{2}(\alpha) \sin (\alpha)} \log \left(1+3 \beta \cos ^{2}(\alpha) \sin (\alpha) \tau\right)
$$

where it is assumed that $\theta(\tau)=0$, and so $\theta(\tau) \sim \log (\tau)$. Taken together, Eqs. (10) and (12) then represent a quasi-circular spiral. From Eq. (10) it can be seen that the rate of change of orbit radius along the spiral can be maximized for an optimum choice of sail pitch angle $\tilde{\alpha}$ such that

$$
\frac{d}{d \alpha}\left(\cos ^{2}(\alpha) \sin (\alpha)\right)=0 \Rightarrow \tan (\tilde{\alpha})=\frac{1}{\sqrt{2}}
$$


This choice of fixed sail pitch angle then maximizes the transverse thrust experienced by the solar sail, where $\tilde{\alpha} \approx 35^{\circ} .{ }^{5}$ However, as will be seen in Section 5 , this choice of fixed pitch angle is no longer optimum for a sail subject to degradation.

\section{Time-dependant optical properties}

The quasi-circular spiral orbit represented by Eqs. (10) and (12) provides a simple approximation to the orbit evolution of a solar sail with a fixed pitch angle and time-invariant optical properties. However, for a sail subject to degradation, the orbit evolution is more complex, but still tractable. Here, the evolution of the sail reflectivity is a function of a time integral of the sail orbit radius, so that the evolution of the orbit radius and reflectivity are clearly strongly coupled. Substituting the time-varying reflectivity from Eq. (3) into Eq. (9) then results in an integro-differential equation, since the reflectivity is an integral function of the accumulated solar radiation exposure such that

$$
\frac{d \xi(\tau)}{d \tau}=2 \beta \cos ^{2}(\alpha) \sin (\alpha) \exp \left(-\frac{\varepsilon}{\omega} \int_{0}^{\tau} \xi\left(\tau^{\prime}\right)^{-2} \cos (\alpha) d \tau^{\prime}\right) \xi(\tau)^{-1 / 2}
$$

where it is assumed that $\eta(0)=1$. This integro-differential equation does not apparently have a closed-form solution, so it is not possible to obtain the sail orbit radius as a function of time directly. However, from Eq. (2) and Eq. (9) it is can be seen that

$$
\frac{d \xi(\tau)}{d \eta(\tau)}=-\frac{2 \omega}{\varepsilon} \beta \cos (\alpha) \sin (\alpha) \xi(\tau)^{3 / 2}
$$

Again assuming that the initial sail reflectivity $\eta(0)=1$, this relationship can then be integrated directly to yield the sail reflectivity as a function of the sail orbit radius as

$$
\eta(\xi(\tau))=1-\frac{\varepsilon}{\lambda}\left(1-\xi(\tau)^{-1 / 2}\right)
$$

where the auxiliary variable $\lambda=\beta \omega \cos (\alpha) \sin (\alpha)$. Then, substituting for the sail reflectivity into Eq. (9), the evolution of the sail orbit radius is defined by 


$$
\frac{d \xi(\tau)}{d \tau}=2 \lambda \cos (\alpha)\left(1-\frac{\varepsilon}{\lambda}\left(1-\xi(\tau)^{-1 / 2}\right)\right) \xi(\tau)^{-1 / 2}
$$

which can be written in more compact form as

$$
\frac{d \xi(\tau)}{d \tau}=A_{1} \xi(\tau)^{-1 / 2}+A_{2} \xi(\tau)^{-1}
$$

and where the collected constants are defined by

$$
\begin{gathered}
A_{1}=2 \lambda(1-\varepsilon / \lambda) \cos (\alpha) \\
A_{2}=2 \varepsilon \cos (\alpha)
\end{gathered}
$$

Re-arranging Eq. (18) it can be seen that

$$
\tau=\int \frac{\xi(\tau) d \xi(\tau)}{A_{1} \sqrt{\xi(\tau)}+A_{2}}+C
$$

which can be integrated directly to yield the solar sail orbit radius as an implicit function of time through

$$
\tau-\frac{2 A_{2}{ }^{2} \sqrt{\xi(\tau)}}{A_{1}{ }^{3}}+\frac{A_{2} \xi(\tau)}{A_{1}{ }^{2}}-\frac{2 \xi(\tau)^{3 / 2}}{3 A_{1}}+\frac{2 A_{2}{ }^{3} \log \left(A_{1} \sqrt{\xi(\tau)}+A_{2}\right)}{A_{1}{ }^{4}}=C
$$

for some constant of integration $C$. This implicit function now defines the evolution of the solar sail orbit radius under the action of sail degradation in closed-form. It can be seen that if $\varepsilon=0$, then Eq. (21) reduces directly to the solution for a quasi-circular spiral with time-invariant optical properties defined by Eq. (10).

The analysis can be extended if required by re-arranging Eq. (16) to obtain $\xi$ as a function of $\eta$ and then substituting into Eq. (2) and integrating to obtain an implicit relationship for $\eta(\tau)$, similar to that obtained for $\xi(\tau)$ through Eq. (21). 
In order to assess the impact of sail degradation, Eq. (16) and Eq. (21) can be used to determine the time evolution of the solar sail orbit radius and reflectivity. These can then be compared to a solar sail with time-invariant optical properties using Eq. (10), and later numerical integration of the exact two-body equations of motion, as will be performed later in Section 5.

\section{Asymptotic limits}

As the solar sail executes a spiral trajectory, its reflectivity is monotonically decreasing due to accumulated exposure to solar radiation. It is therefore expected that the sail will exhibit asymptotic behaviour when it becomes completely absorbing and the transverse component of sail thrust vanishes. Using Eq. (16) the solar sail orbit radius can be written in terms of the sail reflectivity such that

$$
\xi(\eta)=\left(1-\frac{\lambda}{\varepsilon}(1-\eta)\right)^{-2}
$$

In the limit that $\eta \rightarrow 0$ it can be seen from Eq. (22) that the orbit radius has two asymptotic limits corresponding to an outward or inward spiral (with $\alpha>0$ and $\alpha<0$ respectively) such that

$$
\begin{aligned}
& \xi_{\infty}^{+}=\left(1-\frac{|\lambda|}{\varepsilon}\right)^{-2} \\
& \xi_{\infty}^{-}=\left(1+\frac{|\lambda|}{\varepsilon}\right)^{-2}
\end{aligned}
$$

These limits then bound the motion of the solar sail to an annulus, whose inner and outer radii are defined by the sail degradation rate $\varepsilon$, for a given sail lightness number and fixed sail pitch angle.

In order to maximize the outer radius of the annulus it is necessary to choose an optimum fixed sail pitch angle. From Eq. (23a) this new optimum pitch angle $\tilde{\alpha}$ can be determined from 


$$
\frac{d}{d \alpha}\left(1-\frac{\beta \omega \cos (\alpha) \sin (\alpha)}{\varepsilon}\right)^{-2}=0 \Rightarrow \tan (\tilde{\alpha})=1
$$

The optimum fixed sail pitch angle is now a trade-off between maximizing the transverse component of sail thrust while minimizing the projected sail area exposed to the flux of solar radiation. It can be seen from Eq. (13) and (24) that the optimum fixed pitch angle increases from $\tan (\tilde{\alpha})=1 / \sqrt{2}$ for a solar sail with time-invariant optical properties to $\tan (\tilde{\alpha})=1$ for a solar sail subject to degradation. While this is the optimum fixed sail pitch angle in the asymptotic limit to maximize the outer radius of the annulus, the optimum pitch angle for shorter transfers is a function of the sail degradation rate, as will be seen later in Section 5.

In principle these asymptotic limits on the motion of the solar sail could be used as a means of orbit transfer for an entirely passive, low cost solar sail, as noted in Section 1 . The solar sail centre-ofmass and centre-of-pressure can be configured to ensure that a fixed pitch angle is maintained passively. ${ }^{6}$ If the sail film is then engineered to degrade at a certain rate, the asymptotic orbit that the sail winds onto can be chosen a priori, enabling delivery of payloads to given heliocentric orbits. Clearly, such a mode of transfer cannot ensure precision in the final orbit reached, however for space physics missions with simple field and particle instruments this is not a critical issue.

\section{Discussion}

In order to evaluate the utility of the closed-form solutions obtained for a solar sail with time-varying optical properties, comparison with numerical integration can now be made. First, a solar sail with a lightness number of $\beta=0.05$ will be considered with a fixed pitch angle $\tan (\tilde{\alpha})=1 / \sqrt{2}$, first with no sail degradation $(T \rightarrow \infty)$ and then with degradation assuming a half-life of 1 year and 3 years. Such rapid degradation is unlikely for conventional sail films, however a short half-life is used for ease of illustration. Using Eqs. (16) and (21), the evolution of the sail orbit radius is shown in Fig. 2 and the sail reflectivity in Fig. 3 over a duration of 15 years. The numerical integration uses the solar sail two-body problem ${ }^{5}$ with a fixed sail pitch angle, while including sail degradation as defined by 
Eq. (2). It can be seen that the closed-form solutions obtained using the quasi-circular orbit approximation provide an accurate representation of the dynamics of the problem, while clearly missing the orbit eccentricity forcing seen in the numerical integration, as is to be expected.

In order to minimize the time required to reach a given orbit radius, an optimum choice of fixed sail pitch angle needs to be made. For example, using Eq. (21), the time interval required to spiral from $\xi=1$ to $\xi=1.25$ is shown in Fig. 4 for a range of sail degradation half-lives. The optimum fixed sail pitch angle can be determined numerically from a minimization of Eq. (21). For time-invariant optical properties $(T \rightarrow \infty)$ it can be seen that the optimum fixed sail pitch angle corresponds to $\tan (\tilde{\alpha})=$ $1 / \sqrt{2}$, defined by Eq. (13). For a degrading solar sail the optimum fixed sail pitch angle increases. Again, the optimum fixed sail pitch angle is now a trade-off between maximising the transverse component of sail thrust while minimising the projected sail area exposed to the flux of solar radiation. For the asymptotic case, the optimum fixed sail pitch angle to maximise the outer radius of the annulus of motion is given by $\operatorname{an}(\tilde{\alpha})=1$, as discussed in Section 4 . Numerical optimization satisfying exact two-point boundary conditions was performed previously using numerical methods by Dachwald et al. ${ }^{3,4}$

As discussed in Section 4, the time-varying sail reflectivity leads to asymptotic behaviour which can clearly be seen in Fig. 2 for a short sail half-life of 1 year and fixed sail pitch angle $\tan (\tilde{\alpha})=1$. From Eq. (23a) it is found that the outer radius of the annulus limiting the solar sail motion is $\xi_{\infty}^{+}=1.67$ for $T=1$ year (and from Eq. (23b) the inner limit is $\xi_{\infty}^{-}=0.66$ ). This behaviour can be seen in Fig. 5 where the solar sail winds on to an asymptotic orbit of the correct predicted radius. Again, orbit eccentricity forcing can be seen in the numerical integration, as expected. If the sail degradation rate can be engineered, this mode of transfer may allow near circle-to-circle transfer, but with a passive fixed sail pitch angle, as discussed in Section 4.

Finally, while the analysis provides new insights into the behaviour of solar sails subject to degradation, it is expected that a similar analysis may also prove useful to model long duration solar 
electric propulsion spirals with photovoltaic cells subject to accumulated radiation damage. Here the degradation of photovoltaic cells will lead to a reduction in available thrust and hence longer spiral trajectories, particularly for tugs cycling between low Earth orbit and Geostationary orbit. ${ }^{7}$

\section{Conclusions}

The orbit evolution of a solar sail subject to optical degradation has been investigated and an approximate closed-form analytical solution found, assuming a quasi-circular spiral with a fixed sail pitch angle. This new solar sail spiral problem poses challenges since the sail degradation is cumulative, resulting in an integro-differential equation. The solution found provides the time evolution of the solar sail orbit radius in implicit form. It was shown that the closed-form solution provides a good match to numerical integration and that it captures key properties of the dynamics of the problem, including asymptotic limits. The existence of asymptotic limits offers the possibility of near circle-to-circle transfer for an entirely passive solar sail by engineering the degradation rate of the sail film.

\section{Acknowledgments}

This work has been supported by the European Research Council through Advanced Investigator Grant 227571 (VISIONSPACE).

\section{References}

1. Macdonald, M., and McInnes, C. R., "Solar sail science mission applications and advancement," Advances in Space Research, Vol. 48, 2011, pp. 1702-1716.

2. McKay, R., Macdonald, M., Biggs, J., and McInnes, C.R., "Survey of highly non-Keplerian orbits with low thrust propulsion," Journal of Guidance, Control and Dynamics, Vol. 34, No. 3, 2011, pp. 645666. 
3. Dachwald, B., Mengali, G., Quarta, A.A., and Macdonald., "Parametric model and optimal control of solar sails with optical degradation," Journal of Guidance, Control and Dynamics, Vol. 29, No. 5, 2006, pp. $1170-1178$.

4. Dachwald, B., Macdonald, McInnes, C.R., Mengali, G., and Quarta, A.A., “Impact of optical degradation on solar sail mission performance," Journal of Spacecraft and Rockets, Vol. 44, No. 4, 2007, pp. 740-749.

5. McInnes, C.R., Solar sailing: technology, dynamics and mission applications, Springer-Verlag Series in Space Science and Technology, London, ISBN 1-85233-102-X, 1999.

6. Gong, S., Li, J., and Baoyin, H., "Passive stability design for solar sail on displaced orbits," Journal of Spacecraft and Rockets, Vol. 44, No. 5, 2007, pp. 1071-1080.

7. Capadona, L.A., Woytach, J.M., Kerslake, T.W., Manzella, D.H., Christie, R.J., Hickamn, T.A., Scheidegger, R.J., Hoffman, D.J., and Klem, M.D., “Feasibility of large high-powered solar electric propulsion vehicles: Issues and solutions," Paper AIAA 2011-7251, AIAA SPACE 2011 Conference and Exposition, 27-29 September 2011, Long Beach, CA. 


\section{Figure Captions}

Figure 1. Solar sail 2-body problem with orbit radius $r$, polar angle $\theta$ and (fixed) sail pitch angle $\alpha$.

Figure 2. Orbit radius as a function of time for a sail with time-invariant optical properties ( $\mathrm{T} \rightarrow \infty)$ and degrading optical properties, with a sail degradation half-life of 3 years and 1 year, along with corresponding 2-body numerical integration over 15 years (- - - ). Sail lightness number $\beta=0.05$ and fixed sail pitch angle $\tan (\tilde{\alpha})=1 / \sqrt{2}$.

Figure 3. Sail reflectivity as a function of time for a sail with time-invariant optical properties ( $\mathrm{T} \rightarrow$ $\infty)$ and degrading optical properties, with a sail degradation half-life of 3 years and 1 year, along with corresponding 2-body numerical integration over 15 years (- - - -). Sail lightness number $\beta=0.05$ and fixed sail pitch angle $\tan (\tilde{\alpha})=1 / \sqrt{2}$.

Figure 4. Trip time from $\xi=1$ to $\xi=1.25$ for a solar sail with lightness number $\beta=0.05$ and timeinvariant optical properties ( $\mathrm{T} \rightarrow \infty,-$ ) and a sail with degrading optical properties with a sail degradation half-life of 2 years $(---)$. and 1 year $(\cdot-\cdot)$.

Figure 5. Numerical integration of an outward solar sail spiral trajectory over 15 years from an initial circular orbit at $\xi=1$ to an asymptotic orbit at $\xi_{\infty}^{+}=1.67$ with a sail half-life of 1 year. Sail lightness number $\beta=0.05$ and fixed sail pitch angle $\tan (\tilde{\alpha})=1$. Inner asymptotic limit shown at $\xi_{\infty}^{-}=0.66$. 
Figure 1.

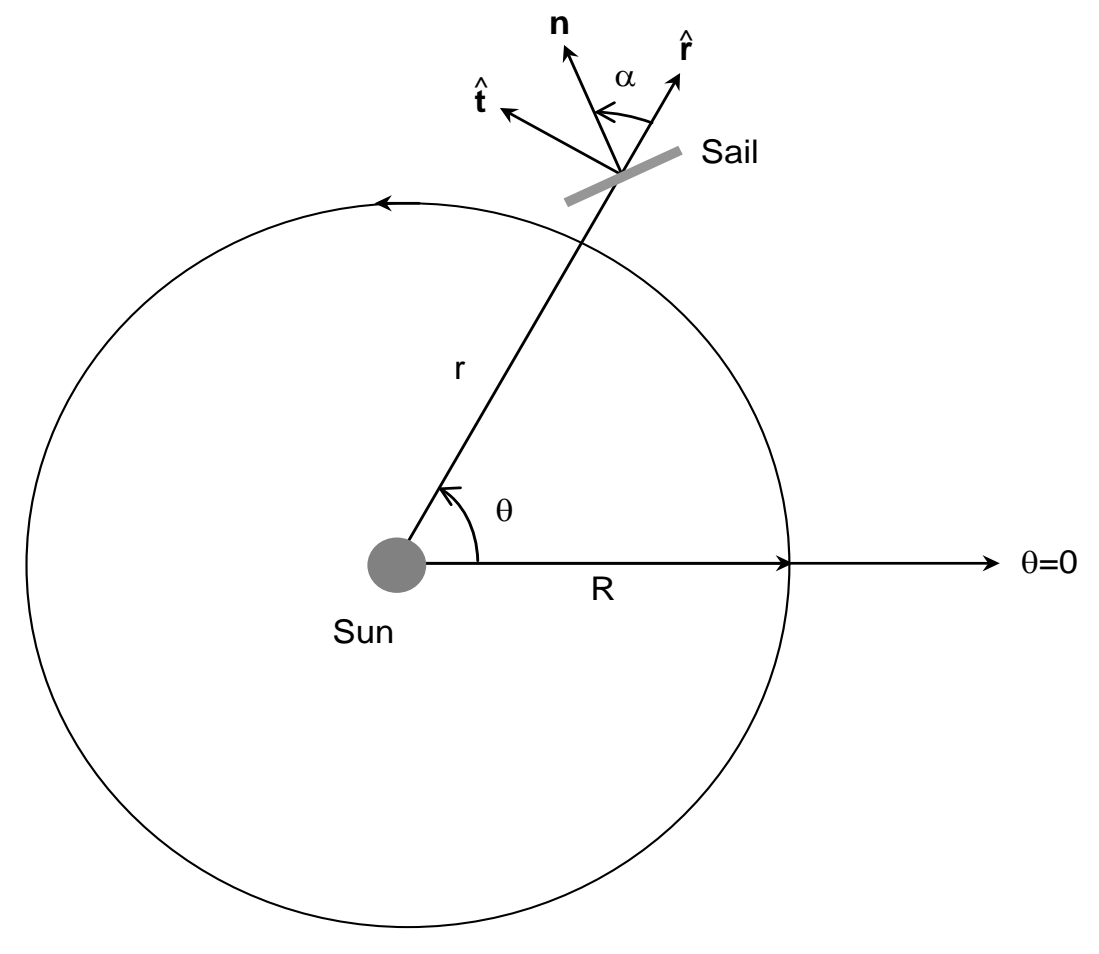


Figure 2.

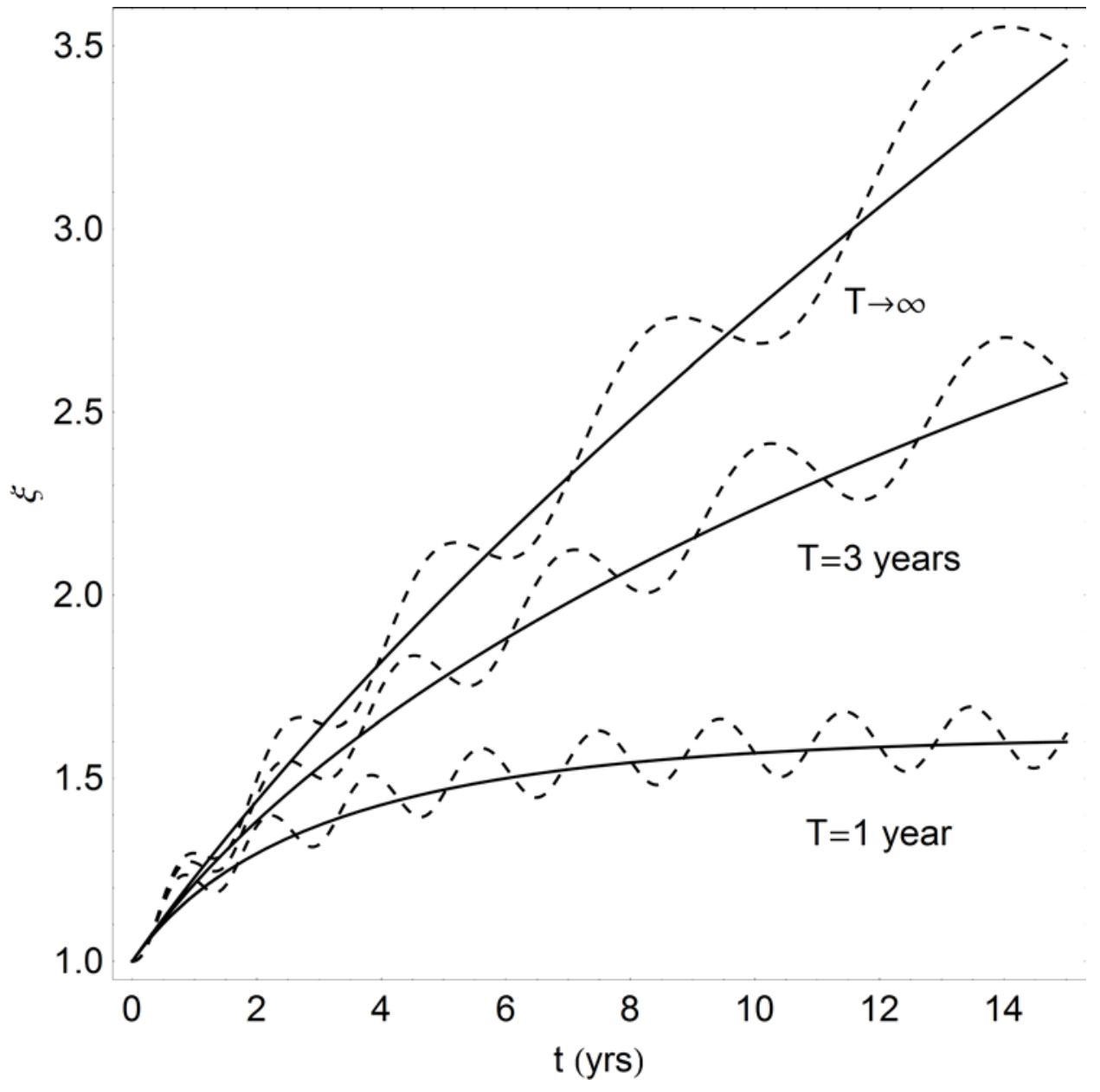


Figure 3.

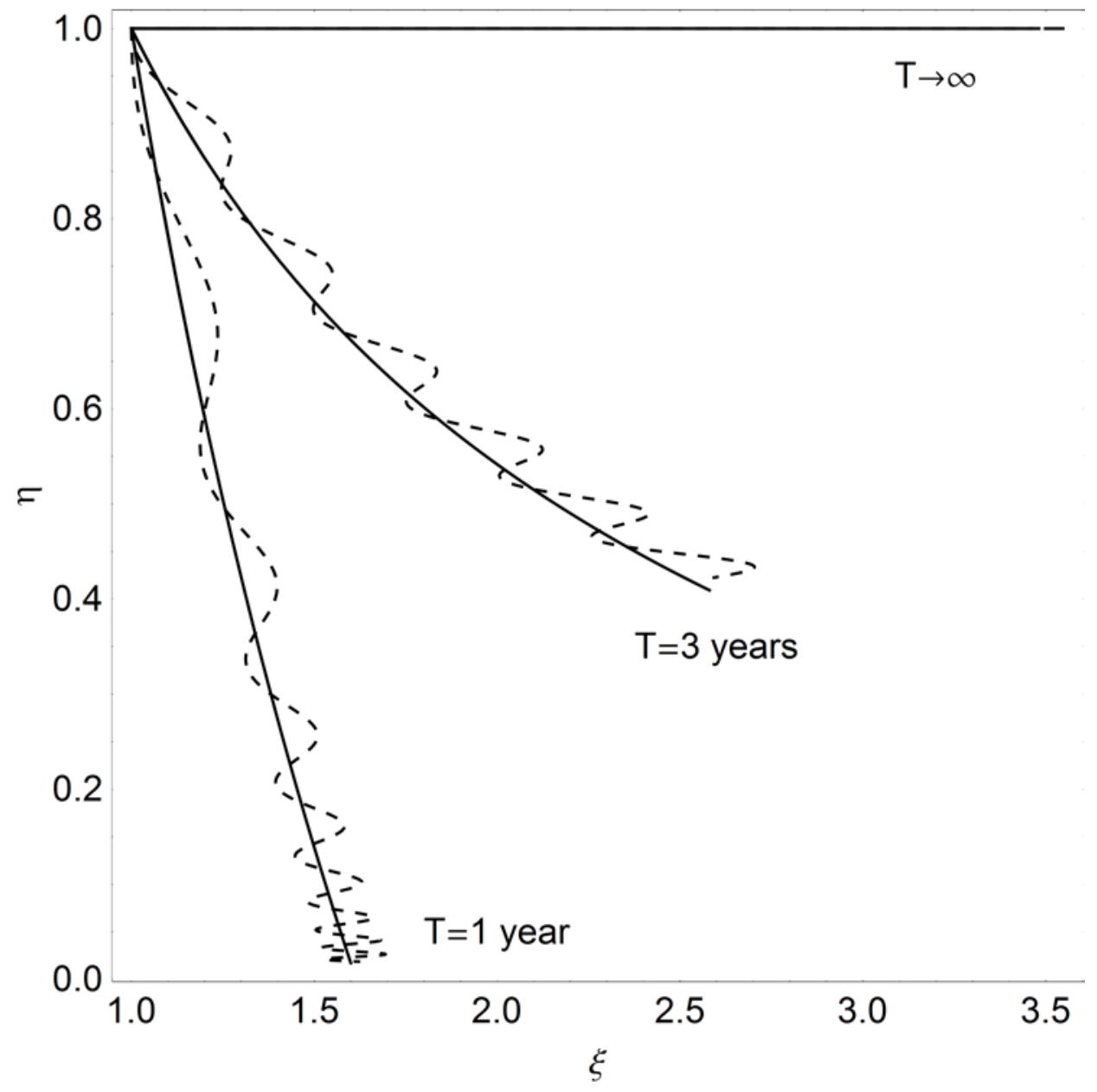


Figure 4.

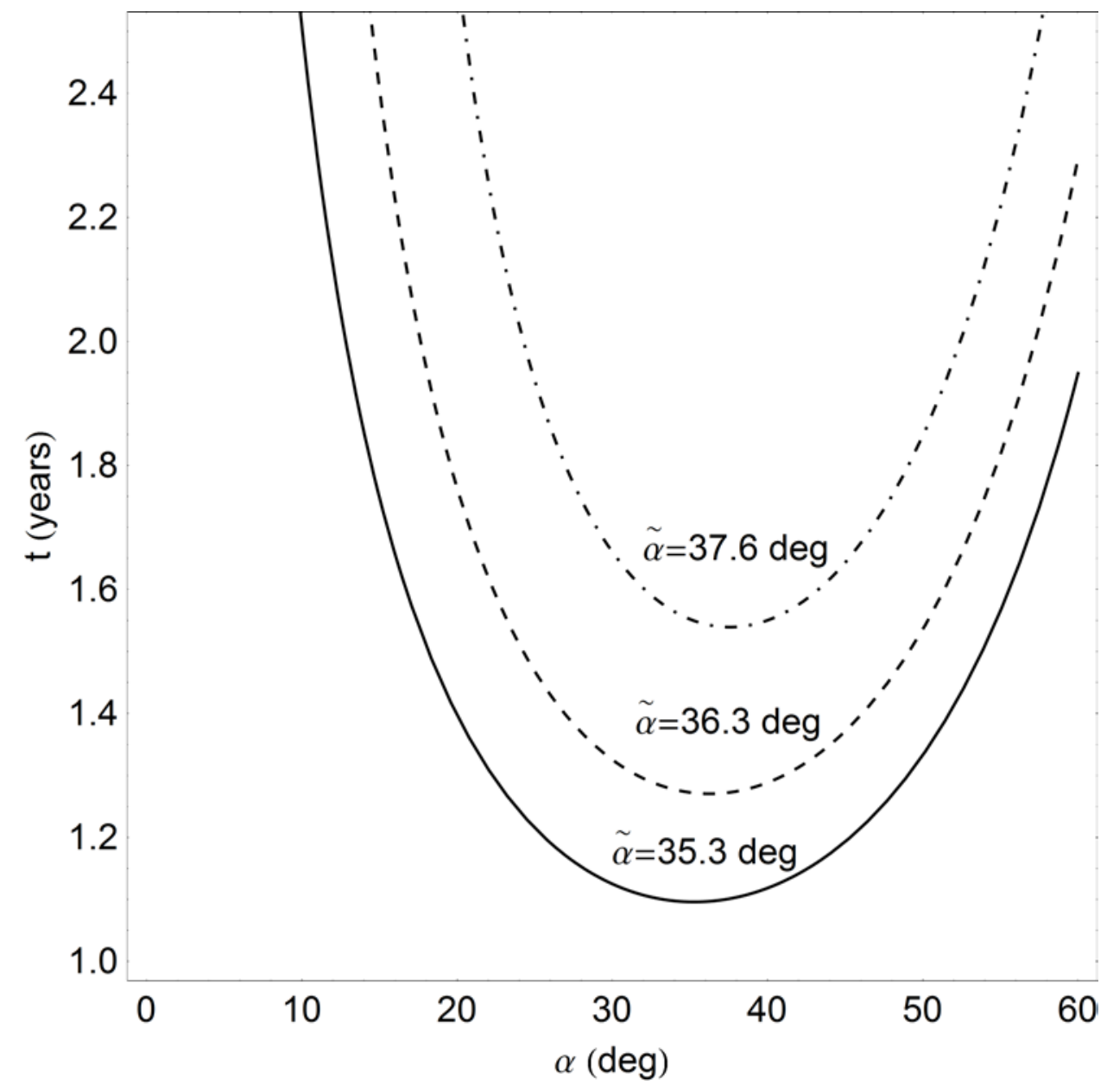


Figure 5.

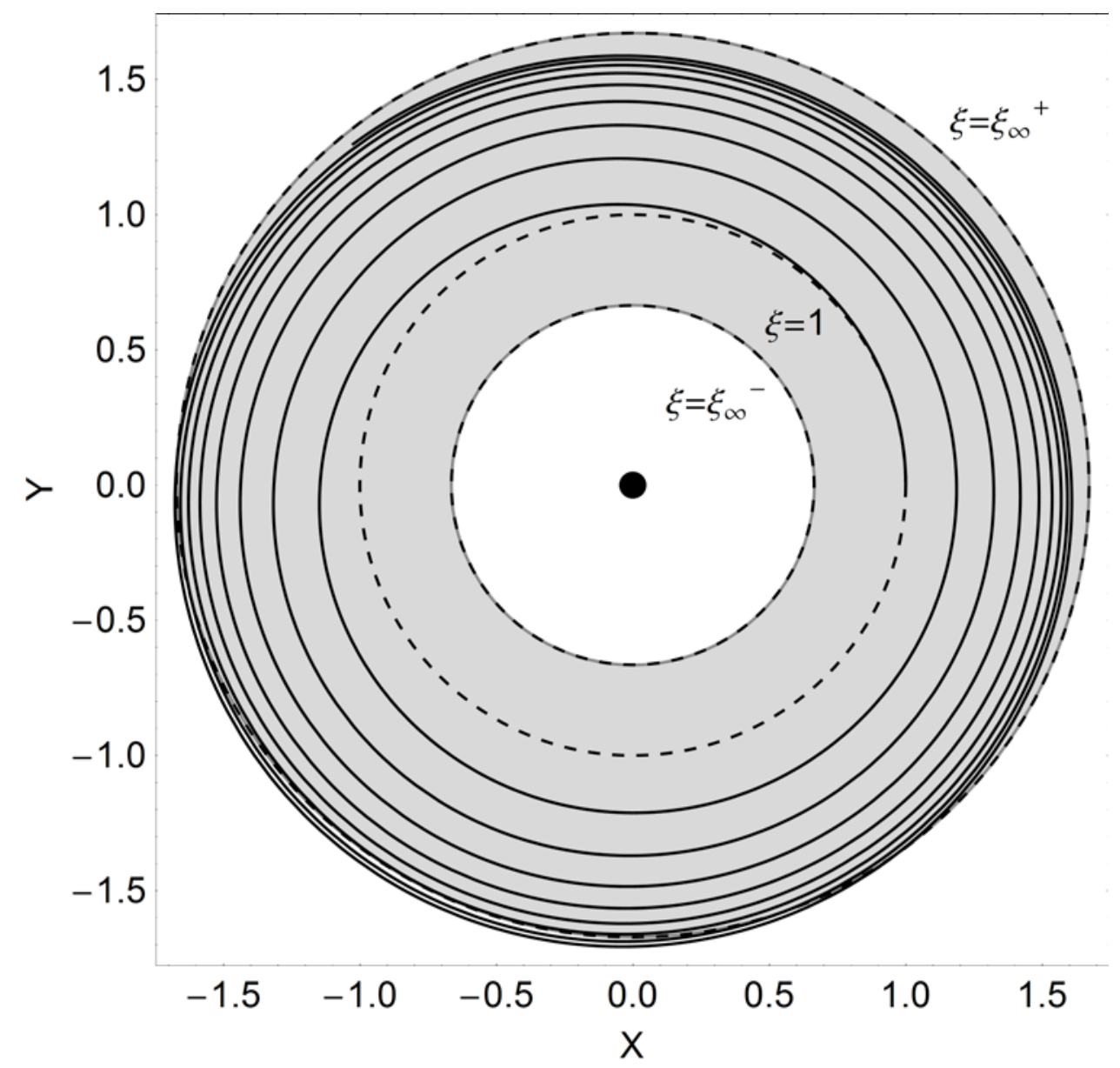

